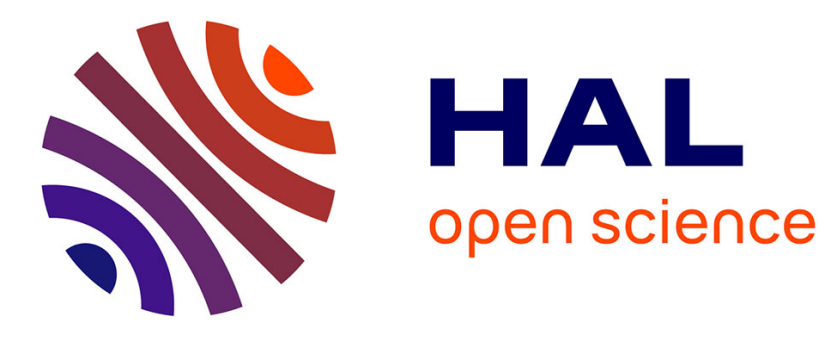

\title{
Noise excess on fractals and percolating systems
}

\author{
R. Rammal
}

\section{To cite this version:}

R. Rammal. Noise excess on fractals and percolating systems. Journal de Physique Lettres, 1984, 45

(21), pp.1007-1014. 10.1051/jphyslet:0198400450210100700 . jpa-00232443

\section{HAL Id: jpa-00232443 \\ https://hal.science/jpa-00232443}

Submitted on 1 Jan 1984

HAL is a multi-disciplinary open access archive for the deposit and dissemination of scientific research documents, whether they are published or not. The documents may come from teaching and research institutions in France or abroad, or from public or private research centers.
L'archive ouverte pluridisciplinaire HAL, est destinée au dépôt et à la diffusion de documents scientifiques de niveau recherche, publiés ou non, émanant des établissements d'enseignement et de recherche français ou étrangers, des laboratoires publics ou privés. 


\title{
LE JOURNAL DE PHYSIQUE-LETTRES
}

J. Physique Lett. 45 (1984) L-1007 - L-1014

1er NOVEMBRE 1984, PAGE $\cdot$ L-1007

Classification

Physics Abstracts

$05.40-05.50-72.90$

\section{Noise excess on fractals and percolating systems}

\author{
R. Rammal (*) \\ Department of Physics, University of Pennsylvania, Philadelphia, P.A., 19104-3859, U.S.A. \\ and \\ Département de Physique, Université de Sherbrooke, Sherbrooke, Québec, J1K 2R1, Canada
}

(Reçu le 13 juillet 1984, accepté le 7 septembre 1984)

\begin{abstract}
Résumé. - Le spectre de bruit $F(\omega)$ des fluctuations de tension d'un réseau self-similaire de résistances est déterminé. Une fonction d'échelle, avec effet de taille finie, valable en toute dimension est proposée pour un milieu désordonné exhibant une diffusion anormale (fréquences aléatoires de saut, désordre de site). Le bruit de basse fréquence est anormal et diverge avec l'exposant $\gamma=1-2 v_{\mathrm{rw}}$ où $v_{\mathrm{rw}}$ désigne l'exposant de la diffusion. Pour les systèmes proches du seuil de percolation, on trouve un excès de bruit et une dépendance intéressante en fréquence.
\end{abstract}

\begin{abstract}
The noise power spectrum $F(\omega)$ of the voltage fluctuations in a self-similar resistor network is determined. A finite-size scaling expression for $F(\omega)$ is given and argued to hold, in all dimensions, for a disordered material exhibiting anomalous diffusion of charged particles (random hopping frequencies, site disordered lattices). The low-frequency noise is anomalous and diverges with the exponent $\gamma=1-2 v_{\mathrm{rw}}$, where $v_{\mathrm{rw}}$ denotes the random-walk exponent for diffusing particles. For percolating networks in the critical region, we find a noise excess and an interesting frequency dependence.
\end{abstract}

1. - Recently, the low-frequency noise fluctuation voltage has been studied near the percolation threshold [1-3]. Measurements were performed in [2] thin Au films (in the range of $7 \mathrm{~nm}$ thick, up to $10 \mathrm{MHz}$ ) and in metal-insulator composites [1] obtained by compacting mixtures of small metal particles (Ag, W) and dielectric powders ( $\mathrm{KCl}, \mathrm{PTFE})$. Far from the percolation threshold, the excess noise seems to show the "usual " $1 / \omega$ frequency dependence. Near percolation the noise spectra changed qualitatively, showing in particular an excess noise at high frequencies.

(*) Permanent address : C.R.T.B.T.-C.N.R.S., B.P. 166X, 38042 Grenoble Cedex, France. 
$1 / \omega$ fluctuations have been found in a great many physical systems [4]. However, the statistical mechanical foundation of $1 / \omega$ noise has not been fully established and, there is so far no convincing explanation for the "universality " of this phenomenon. The purpose of this Letter is not to attempt a new theoretical approach to obtain the $1 / \omega$ spectrum. Instead we shall present new results pertaining to a simple model of resistor networks. The physical model is equivalent to the diffusion process in the considered structure. Anomalous diffusion is actually known to occur in various systems : self-similar structures, disordered materials, etc... Therefore, we are interested in equilibrium noise, whose occurrence in macroscopic bodies is due to their atomic-molecular structure. The basic result of the paper (Eqs. (3) and (4)) is the occurrence of a singular low-frequency noise fluctuation voltage with a spectrum $F(\omega) \sim \omega^{-\gamma}$ where $\gamma=1-2 v_{\mathrm{rw}}, v_{\mathrm{rw}}$ is the anomalous diffusion exponent on the structure in question. A finite size scaling expression for $F(\omega)$ is proposed and argued to hold in various situations. Near the percolation threshold, we find a noise excess over the perfect network noise and an interesting frequency dependence.

2. - The model we consider is a resistor network on a lattice. Nearest neighbour sites of the lattice are connected by conductors each of which is chosen to have a purely resistive impedance $R$ (conductance $\sigma=1 / R$ ). Each site not belonging to the electrodes is connected to ground by a circuit consisting of a capacitance $C$ and an $\operatorname{emf} U_{i} \mathrm{e}^{i \omega t}$. The emf on the electrode is equal to the external potential (Fig. 1).

Noise is introduced into the network in the following way. In each conductor between nn sites $k, l$ there is a random, thermally generated component to the current $J_{k l}(t)$ with zero mean and a correlation function $\left\langle J_{k l}(t) J_{k l}\left(t^{\prime}\right)\right\rangle=2 k_{\mathrm{B}} T \sigma \delta\left(t-t^{\prime}\right)$ where $T$ is the temperature. The noise current in each conductor is independent of that in other conductors, and $J_{k l}(t)=-J_{l k}(t)$. Two large planar electrodes, separated by a distance $L$ (length scale of the circuit) along the $x$ axis are connected to the network and the voltage power spectrum measured between these two electrodes. As usual the power spectrum of the voltage difference $V$ across the electrodes is defined by

$$
S_{V}(\omega)=\lim _{\theta \rightarrow \infty} \frac{1}{\theta}\left|\int_{0}^{\theta} \mathrm{e}^{i \omega t} V(t) \mathrm{d} t\right| .
$$

Note that other power spectra can be deduced from $S_{V}(\omega)$, through the known relation $S_{I}(\omega) / I^{2}=S_{R}(\omega) / R^{2}=S_{V}(\omega) / V^{2}$ where $I$ and $R$ denote the total current and the resistance of the network, respectively.

The basic equation of the considered network is given by the conservation of charge $Q_{i}$ at each site $i$

$$
\frac{\mathrm{d} Q_{i}}{\mathrm{~d} t}=-\frac{1}{R} \sum_{k}\left(V_{i}-V_{k}\right)-\sum_{k} J_{i k}(t)
$$

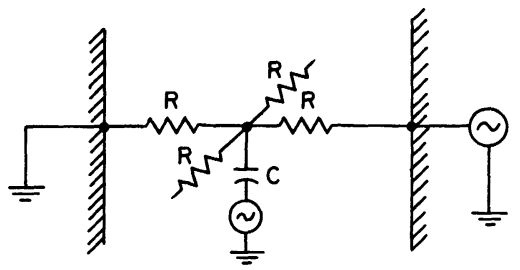

Fig. 1. - Typical circuit configuration. 
where $V_{i}$ is the voltage at site $i . Q_{i}$ is related to $V_{i}$ by $Q_{i}=C\left(V_{i}-U_{i}\right)$. Equation (1) is a Langevintype equation where the fluctuation is introduced by adding a random driving source term $\{J(t)\}$ to the right hand side of the diffusion equation. Obviously, the nature of the diffusion field is irrelevant and the results pertain to carrier diffusion, heat conduction, etc.

The determination of the noise spectra arising from equation (1) can be carried out using Green's function techniques [5]. In general the power spectrum is governed by : the diffusion kernel associated with (1), the spectra of the source terms and the boundary conditions. The expansion of the Green's function in normal modes leads to a simple expression of $S_{V}(\omega)$ as a sum over the spectrum of the linear operator appearing in equation (1).

3. - Let us consider first the case of a regular lattice, of linear size $L$ containing $L^{d}$ nodes. The eigenmodes in this case are plane waves. The spectra of the source terms are assumed to be white and spatially uncorrelated. The expression of $S_{V}(\omega)=F(\omega)$ is therefore easy to derive (see Appendix for explicit calculation at $d=1$ ). In general, the result takes the following simple form

$$
F(\omega)=\frac{2 k_{\mathbf{B}} T}{G(L)} \psi(l(\omega) / L)
$$

Here, we have introduced the diffusion length $l(\omega)=(2 D / \omega)^{1 / 2}$, where $D=a^{2} / R C$ is the diffusion constant ( $a=$ lattice spacing) and $G(L)$ denotes the macroscopic conductance of the network. Using Einstein's relation, $F(\omega)$ can also be written as

$$
F(\omega)=\frac{A}{D L^{d-2}} \psi(l(\omega) / L)
$$

where $A$ denotes a constant term. In equations (2). $\psi$ denotes a scaling function, with $\psi(u \ll 1) \simeq u$ and $\psi(u \gg 1) \simeq 1$.

As expected, Nyquist's result is recovered at low frequency :F( $\omega) \simeq L^{-d+2} / D$ independent of $\omega$. The crossover to a diffusion regime : $F(\omega) \simeq \omega^{-1 / 2}$ occurs at $\omega=\omega_{D} \equiv \omega_{0}(a / L)^{2}$, where $\omega_{0}=1 / R C$. For $\omega \gg \omega_{0}, l(\omega) \ll a$ and $F(\omega) \simeq \omega^{-2}$ (not described by Eqs. (2)). The physical origin of these different regimes can be explained as follows :

a) $\omega \ll \omega_{D}$ : in this limit the fluctuations become correlated across the entire sample. The space-time correlation function of $V(\mathbf{r}, t)$ becomes independent of $\omega$ as $\omega \rightarrow 0$.

b) $\omega_{D} \ll \omega \ll \omega_{0}: l(\omega) \ll L$ and the sample may be divided into correlated regions each of linear size $l(\omega)$. However only the two thin plates of width $l(\omega)$ located at the electrodes can contribute to $F(\omega)$. Therefore in the previous expression $L^{d-2}$ must be replaced by the conductance of these outer shells $\sim L^{d-1} / l(\omega)$ and the above result follows.

c) $\omega \gg \omega_{0}$ : at very high frequencies where $l(\omega)$ becomes smaller than the lattice spacing $a$, the capacitors become short-circuits to ground. $L^{d-2}$ must be replaced by $L^{d-1} / a$ as in (b) and $F(\omega)$ is given by

$$
F(\omega) \simeq\left(\frac{\omega_{0}}{\omega}\right)^{2} \frac{a}{D L^{d-1}}
$$

Before considering the case of fractal networks, it is interesting to remark that all the previous analysis can be used to calculate $F(\omega)$ in the case of disordered $R C$ circuits. Only $l(\omega)$ and then $D(\omega) \sim \omega l^{2}(\omega)$ are to be modified. In general, $l(\omega) \sim \omega^{-v_{\mathrm{rw}}}$ at low frequencies, where $v_{\mathrm{rw}}$ denotes the anomalous diffusion exponent $\left(v_{\mathrm{rw}}=\frac{1}{2}\right.$ for ordered regular lattices $)$. Anomalous diffusion due to disorder leads to a frequency dependent diffusion coefficient $D(\omega) \sim \omega^{1-2 v_{\mathrm{rw}}}$ and nontrivial cases where $v_{\mathrm{rw}}<\frac{1}{2}$ have been investigated in great details in reference [6]. In our circuit, 
disorder can be introduced either in $R$ 's or in $C_{i}$ 's. Both models lead to an equivalent diffusion problem, with a distribution $\rho(W)$ of hopping rates $(W)$ between nearest neighbouring sites. For $d>1$, similar results [7] can be derived for site disorder with a probability distribution of the mean waiting time $\left(\sim R C_{i}\right)$ at the lattice sites.

Our previous analysis leads to the following prediction for $F(\omega)$

$$
F(\omega) \simeq \begin{cases}1 / L^{d-2} \omega^{1-2 v_{\mathrm{rw}}}, & l(\omega) \ll L \\ 1 / L^{d-1} \omega^{1-v_{\mathrm{rw}}}, & l(\omega) \gg L\end{cases}
$$

the expression of $v_{\mathrm{rw}}\left(\operatorname{Refs}\right.$. [6, 7]) is given by : $v_{\mathrm{rw}}=\frac{1}{2}$ for classes $(\mathrm{a})\left(\left\langle W^{-1}\right\rangle\right.$ and higher moments finite) and (b) $\left(\rho(W \sim 0)=\right.$ const) and, $v_{\mathrm{rw}}=\left[2+d /\left(\frac{1}{\alpha}-1\right)\right]^{-1}$ for class (c) where $\rho(W)=(1-\alpha) W^{-x}, 0 \leqslant W \leqslant 1,0<\alpha<1$. The result of equation (3) is particularly interesting because of the divergence of $F(\omega)$ at low frequencies : $F(\omega) \sim \omega^{-1+2 v_{\mathrm{rw}}}$ for class (c). This regime is absent in the Euclidean regular case as well as for classes (a) and (b). Equation (3) generalizes the $d=1$ result of reference [8] to all $d$. Furthermore, it should be noted that in both cases, the exponents of $F(\omega)$ are smaller than 1 , for all $d$ and all $\alpha$. The $1 / \omega$ spectrum is only reached for $\alpha \lesssim 1$ or $d \alpha \gg 1$.

4. - Let us consider now the case of a fractal lattice, where anomalous diffusion is known to occur, without any disorder [9]. The exponent $v_{\mathrm{rw}}$ characterizing this anomalous diffusion is given by the ratio $v_{\mathrm{rw}}=\tilde{d} / 2 \bar{d}$ of the spectral $(\tilde{d})$ and fractal $(\bar{d})$ dimensions $[10,11]$. As above, three regimes, according to the relative values of $l(\omega) / L$ and $l(\omega) / a$ are to be considered (Fig. 2).

a) $l(\omega) \gg L$ : the previous argument holds and

$$
F(\omega)=\frac{A}{L^{\bar{d}-2} D(\omega)} \sim 1 / L^{\bar{d}-2} \omega^{1-2 v_{\mathrm{rw}}} ; \text { i.e. } \gamma=1-2 v_{\mathrm{rw}}
$$

Note that (4) is independent of the contact geometry between the electrodes and the sample.

b) $a \ll l(\omega) \ll L$ : the $\omega$ and $L$ dependence of $F(\omega)$ is controlled by the nature of contact, i.e. the conductance of the outer shell of width $l(\omega)$. For a homogeneous sharp boundary, the conductance is given by $l(\omega) / L^{\bar{d}-1}$, leading to $F(\omega) \sim 1 / L^{\frac{d}{d}-1} \omega^{1-v_{r w}}$. Otherwise when contacts are made with a small set of nodes, both the $L$ and $\omega$ dependences may be modified. In any case, the conductance of a sample of linear size $L$ is given by a scaling function $G(L) \sim L^{\beta_{100}} f(l(\omega) / L)$.

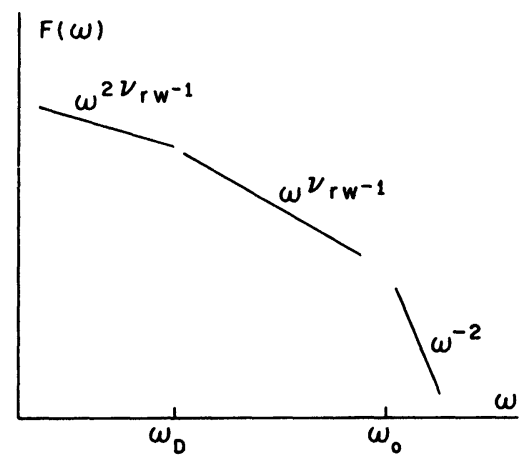

Fig. 2. - Predicted power spectrum of the equilibrium voltage fluctuations in a resistor network. Here $v_{\mathrm{rw}}{ }^{\circ}$ denotes the diffusion exponent. 
Depending on the nature of contact with electrodes, the expression of $F(\omega)$ must be modified by taking into account this scaling behaviour (here $\beta_{\mathrm{loc}}=\bar{d}-1 / v_{\mathrm{rw}}$, See Ref. [11]).

c) $l(\omega) \ll a:$ as in case b) the $L$ dependence is governed by the contact geometry, but the frequency dependence is given trivially by $F(\omega) \sim\left(\omega_{0} / \omega\right)^{2}$.

It should be noted that, at low frequency $(l(\omega) \gg L)$, the same argument can be used to calculate the power spectra of other fluctuating quantities. For instance, if we are interested in the spatial average of the diffusing field (charge $Q$, carrier concentration $N$, etc...), the result we obtain is $S_{N}(\omega) \sim \omega^{-\gamma}$ where $\gamma=1-\tilde{d} / 2(\tilde{d}<2)$ and $\gamma=0(\tilde{d}>2)$. This frequency dependence must be contrasted with equations (3) and (4) where the fractal dimension $\bar{d}$ appears through $v_{\mathrm{rw}}$. The origin of this difference must be traced back to the expression of the considered fluctuating quantity.

5. - We turn to the case of the percolating networks at $\Delta p=p-p_{\mathrm{c}} \gtrsim 0$. In this case four lengths are of importance : the lattice spacing $a$, the diffusion length $l(\omega)$, the percolation correlation length $\xi_{p}=a .(\Delta p)^{-v_{p}}$ and the sample linear size $L$. Different regimes can be classified according to the ratio $\xi_{p} / L$. In the following we shall limit our discussion to the relevant physical regimes. Cases where $\omega \gg \omega_{0}$ are somewhat trivial and are not discussed.

5.1 CASE $\xi_{p} / L \ll 1$. - The sample can be viewed as a Euclidean lattice, with unit cells containing $\xi_{p}^{d}$ sites each. The expression of $F(\omega)$ can then be deduced from equations (2) by rescaling the unit length $a$ by a factor $\xi_{p} / a: a \rightarrow \xi_{p}$ and the frequency $\omega \rightarrow \omega^{*}=\omega \xi_{p}^{1, v_{r w}}$, where $[10,11]$ $v_{\mathrm{rw}}=v_{p} /\left(t-\beta_{p}+2 v_{p}\right)$. The renormalized diffusion length becomes $l^{*}(\omega) \simeq \xi_{p} \omega^{*-1 / 2}$.

i) $l^{*}(\omega) \gg \xi_{p}$ : in this limit, the diffusion length is larger than the lattice « spacing " $\left(\xi_{p}\right)$ and two cases must be distinguished.

a) $l^{*}(\omega) \gg L \gg \xi_{p}: \omega^{* 1 / 2} \ll \frac{\xi_{p}}{L} \ll 1$ corresponds to the low frequency regime of Euclidean lattices. $F(\omega)$ takes therefore the following form

$$
F(\omega)=\frac{A}{L^{d-2} D(p)} P(p) \sim \frac{A}{L^{d-2}}(\Delta p)^{2_{p}-t} .
$$

Here $P(p) \sim(\Delta p)^{\beta_{p}}$ denotes the probability of being on the infinite cluster (2 electrodes) and $D(p) \sim a^{2} \omega_{0}(\Delta p)^{t-\beta_{p}}$ is the diffusion constant.

b) $\xi_{p} \ll l^{*}(\omega) \ll L: 1 \gg \omega^{* 1 / 2} \gg \xi_{p} / L$ and as above

$$
F(\omega)=\frac{A \cdot P(p)}{L^{d-2} D(p)} \cdot \frac{l^{*}(\omega)}{L} .
$$

These two regimes can be summarized into a scaling form (Eqs. (2)) :

$$
F(\omega)=A \frac{P(p)}{L^{d-2} D(p)} \psi\left(l^{*}(\omega) / L\right) .
$$

ii) $l^{*}(\omega) \ll \xi_{p}$ : in this case $\omega^{* 1 / 2} \gg 1$ and $F(\omega)$ is given by

$$
F(\omega)=A\left(\frac{\omega_{0}}{\omega^{*}}\right)^{2} \frac{\xi_{p}}{L} \frac{P(p)}{L^{d-2} D(p)} .
$$

These results (Eqs. (5)-(8)) extend in a transparent way the meanfield calculations of reference [12]. Note however the difference between the two results in the case i-a) where instead of a logarithmic divergence : $F(\omega) \simeq\left[\ln \frac{16 \xi_{p} / L^{2}}{\omega^{*}}\right]^{2}$ we find a saturation regime (Eq. (5)). 
Very close to $p_{\mathrm{c}}$ the noise is proportional to $\omega^{-1 / 2}$ (classical diffusion) down to a frequency given by $\omega^{*} / \omega_{0} \gtrsim \xi_{p} / L$, i.e. $\omega \gtrsim \omega_{0}\left(\frac{a}{L}\right)^{2}(\Delta p)^{t-\beta_{p}}$ instead of $\omega_{0}(a / L)^{2}$ in the perfect network (Eqs. (2)). This can be viewed as a high frequency noise excess and should be compared with the experimental data. However the origin of this noise excess is somewhat trivial, resulting from the renormalization of the diffusion coefficient $D(p)=D(p=1) . .(\Delta p)^{t-\beta_{p}}$ close to $p_{c}$.

5.2 CASE $\xi_{p} / L \gg 1$. - Very close to $p_{\mathrm{c}}$ the correlation length $\xi_{p}$ becomes larger than the linear size of the sample.

In this limit, anomalous diffusion takes place and the fractal nature of the infinite cluster becomes relevant. The diffusion length is given by $l(\omega)=a\left(\omega / \omega_{0}\right)^{-v_{\mathrm{rw}}}$ and the diffusion coefficient $D(\omega) \simeq \omega^{1-2 v_{\mathrm{rw}}}$. The discussion above leads to the predictions

a)

$$
\begin{aligned}
& l(\omega) \gg L: \quad F(\omega)=\frac{A}{L^{\bar{d}-2} D(\omega)} P(p) \\
& l(\omega) \ll L: \quad F(\omega)=\frac{A}{L^{\bar{d}-2} D(\omega)} \frac{l(\omega)}{L} P(p)
\end{aligned}
$$

where $\bar{d}=d-\beta_{p} / \nu_{p}$ denotes the fractal dimension of the percolating clusters. The numerical values of the exponents $\gamma$ in equations (9) and (10) are respectively $[10,11]$ :

- for $d=6: d=4 / 3, \bar{d}=4$ and $v_{\mathrm{rw}}=\frac{1}{6}$ leading to $\gamma^{\prime}=1-v_{\mathrm{rw}}=\frac{5}{6}$ and $\gamma=1-2 v_{\mathrm{rw}}=\frac{2}{3}$

- for $d=2: \hat{d} \lesssim 4 / 3, \bar{d} \simeq 1.9$ and $\gamma^{\prime} \simeq 0.65, \gamma \simeq 0.3$.

6. - The anomalous low-frequency noise obtained in this paper stems' from the anomalous diffusion in various systems : fractals, disordered materials, percolating networks, etc. The predicted power spectra exponents $\left(\gamma\right.$ and $\left.\gamma^{\prime}\right)$ are definitely smaller than one, in comparison with $1 / \omega$ noise. The analysis of the experimental data should give us a clearcut test of the relevance of this diffusion model.

The model discussed in this paper is probably appropriate for evaporated metal-insulator mixtures. For electronic systems, like metals (e.g. $\mathrm{Cu}, \mathrm{Ag}, . .$.$) a new length : the screening length \kappa^{-1}$ must be taken into account. In this case $\kappa^{-1}$ introduces a new cut off in the spectrum and the results discussed above are recovered only at $\kappa L \ll 1$. With screening effect, the expression of $F(\omega)$ is modified (see Eq. (A.10), Appendix) and for $\kappa L \gg 1: F(\omega) \sim 1 /\left(\omega^{2}+D \kappa^{4}\right.$ ) which reduces to Johnson noise at $\omega=0$. The condition $\kappa L \ll 1$ is impossible to achieve in practice for pure metals. The investigation of $F(\omega)$ in the case of disordered metals would be the next step in this program.

\section{Acknowledgments.}

This work was supported in part by U.S. National Science Foundation under Grant No. DMR 8219216. The author would like to thank Dr. C. Tannous and Dr. A. M. S. Tremblay for friendly conversations. The author also would like to thank the Physics Department of the University of Pennsylvania and the Département de Physique de l'Université de Sherbrooke, where this work was done, for their hospitality. 


\section{Appendix}

In this appendix we shall illustrate the main steps leading to equations (2), in one dimension. The voltage on the $i$ th node obeys the Langevin equation

$$
C \frac{\mathrm{d} V_{i}}{\mathrm{~d} t}=\frac{1}{R}\left(V_{i+1}+V_{i-1}-2 V_{i}\right)+\left(J_{i, i+1}-J_{i, i-1}\right) .
$$

A similar equation is obeyed by $Q_{i}$. In the limit where the lattice spacing $a$ becomes very small, $V$ becomes a continuous function of position and time $V=V(x, t)$ and obeys the diffusion equation

$$
\frac{\partial V}{\partial t}=D \frac{\partial^{2} V}{\partial x^{2}}+\frac{1}{c} \frac{\partial J}{\partial x}
$$

where $D=\lim _{a \rightarrow 0} \frac{a^{2}}{R C}$ is the diffusion coefficient and $c=\lim _{a \rightarrow 0} \frac{C}{a}$ is the capacitance per unit length. Let us denote by $V(k, \omega)$ the Laplace-Fourier transform of $V(x, t)$ :

$$
V(x, t)=\int_{-\infty}^{\infty} \mathrm{d} k \int_{-\infty}^{\infty} \frac{\mathrm{d} \omega}{2 \pi} \mathrm{e}^{i k x} \mathrm{e}^{-i \omega t} V(k, \omega) .
$$

It is easy to derive the following expression for $V(k, \omega)$ :

$$
V(k, \omega)=\frac{i k . J(k, \omega) / c}{D k^{2}-i \omega}
$$

The quantity of interest is the voltage difference between two sites at distance $L$

$$
\theta(L) \equiv V(L)-V(0)=\int_{0}^{L} \frac{\partial V}{\partial x}(x, t) \mathrm{d} x
$$

and its Fourier transform

$$
\theta(\omega)=\int_{0}^{L} \frac{\mathrm{d}}{\mathrm{d} x} V(x, \omega) \mathrm{d} x
$$

where

$$
V(x, \omega)=(2 \pi)^{-1 / 2} \int_{-\infty}^{\infty} \mathrm{d} k \mathrm{e}^{i k x} V(k, \omega)
$$

Then

$$
\theta(\omega)=(2 \pi)^{-1 / 2} \int_{0}^{L} \mathrm{~d} x \int_{-\infty}^{\infty} \mathrm{d} k \cdot i k V(k, \omega) \mathrm{e}^{i k x}
$$

The integration over $x$ yields

$$
\theta(\omega)=(2 \pi)^{-1 / 2} \int_{-\infty}^{\infty} \mathrm{d} k \frac{i k \cdot J(k, \omega) / c}{D k^{2}-i \omega}\left(\mathrm{e}^{i k L}-1\right) .
$$


Using $\left\langle J(k, \omega) J^{*}\left(k^{\prime}, \omega\right)\right\rangle=2 k_{\mathrm{B}} T \sigma \delta\left(k-k^{\prime}\right) / 2 \pi$, we get

$$
F(\omega) \equiv\left\langle\theta(\omega) \theta^{*}(\omega)\right\rangle=\frac{4}{\pi} A \int_{-\infty}^{\infty} \mathrm{d} k \frac{D k^{2} \cdot \sin ^{2}(k L / 2)}{\left(D k^{2}\right)^{2}+\omega^{2}}
$$

Here $A=k_{\mathrm{B}} T\left(\frac{R}{a}\right) D$ denotes a constant term.

Integration over $k$ gives [8]

$$
F(\omega)=\frac{A}{D} l(\omega)\left[1-\mathrm{e}^{-L / l(\omega)}\left(\cos \frac{L}{l(\omega)}-\sin \frac{L}{l(\omega)}\right)\right]
$$

where $D=a^{2} / R C$ and $l(\omega)=(2 D / \omega)^{1 / 2}$ is the diffusion length. For $l(\omega) \ll L, F(\omega) \sim \omega^{-1 / 2}$ (diffusion noise) and for $l(\omega) \gg L$ the Nyquist's result $F(\omega)=2 k_{\mathrm{B}} T$. $R$ is recovered ( $\mathcal{R}=$ total resistance). The limit $l(\omega) \ll a$ is somewhat trivial, but not described by the continuum approach (Eq. (A.2)). In this region $\omega \gg \omega_{0}\left(\omega_{0}=\frac{1}{R C}\right)$ all capacitors become virtual short-circuits to ground and all dimension dependent effects then disappear. This single $R C$ circuit gives the known behaviour $F(\omega) \simeq 2 k_{\mathrm{B}} T R /\left(1+\omega^{2} / \omega_{0}^{2}\right) \sim \omega^{-2}$.

\section{References}

[1] Rudman, D. A. and Garland, J. C., Bull. Am. Phys. Soc. 29 (1984) 352.

[2] Laibowitz, R. B., Voss, R. F., Gefen, Y., Koch, R. H., Viggiano, J. M. and Allessandrini, E. I., Bull. Am. Phys. Soc. 29 (1984) 352.

[3] Clerc, J. P., private communication.

[4] Dutta, P. and Horn, P. M., Rev. Mod. Phys. 53 (1981) 497, and references therein.

[5] VAN Vliet, K. M. and Fassett, J. R., Fluctuation phenomena in Solids, Ed. by R. W. Burgers (Academic Press, New York) 1965.

[6] Alexander, S., Bernasconi, J., Schneider, W. R. and Orbach, R., Rev. Mod. Phys. 53 (1981) 175.

[7] Machta, J., Preprint (1984).

[8] Kariotis, R. and Stephen, M. J., Phys. Lett. A 93 (1983) 349.

[9] Rammal, R., in Third Conference on Fractals : "Fractals in the Physical Sciences " to appear in J. Stat. Phys. (1984).

[10] Alexander, S. and Orbach, R., J. Physique Lett. 43 (1982) L-623.

[11] Rammal, R. and Toulouse, G., J. Physique Lett. 44 (1983) L-13.

[12] Stephen, M. J., J. Phys. C. 11 (1978) L-965. 